\title{
A comparison of event-related potentials of humans and rats elicited by a serial feature-positive discrimination task
}

Citation for published version (APA):

Sambeth, A., \& Maes, J. H. R. (2006). A comparison of event-related potentials of humans and rats elicited by a serial feature-positive discrimination task. Learning and Motivation, 37, 269-288. https://doi.org/10.1016/j.Imot.2005.06.001

Document status and date:

Published: 01/01/2006

DOI:

10.1016/j.Imot.2005.06.001

Document Version:

Publisher's PDF, also known as Version of record

\section{Document license:}

Taverne

Please check the document version of this publication:

- A submitted manuscript is the version of the article upon submission and before peer-review. There can be important differences between the submitted version and the official published version of record.

People interested in the research are advised to contact the author for the final version of the publication, or visit the DOI to the publisher's website.

- The final author version and the galley proof are versions of the publication after peer review.

- The final published version features the final layout of the paper including the volume, issue and page numbers.

Link to publication

\footnotetext{
General rights rights.

- You may freely distribute the URL identifying the publication in the public portal. please follow below link for the End User Agreement:

www.umlib.nl/taverne-license

Take down policy

If you believe that this document breaches copyright please contact us at:

repository@maastrichtuniversity.nl

providing details and we will investigate your claim.
}

Copyright and moral rights for the publications made accessible in the public portal are retained by the authors and/or other copyright owners and it is a condition of accessing publications that users recognise and abide by the legal requirements associated with these

- Users may download and print one copy of any publication from the public portal for the purpose of private study or research.

- You may not further distribute the material or use it for any profit-making activity or commercial gain

If the publication is distributed under the terms of Article 25fa of the Dutch Copyright Act, indicated by the "Taverne" license above, 


\title{
A comparison of event-related potentials of humans and rats elicited by a serial feature-positive discrimination task
}

\author{
A. Sambeth ${ }^{\mathrm{a}, \mathrm{b}, *}$, J.H.R. Maes ${ }^{\mathrm{a}}$ \\ ${ }^{a}$ Nijmegen Institute for Cognition and Information/Department of Biological Psychology, \\ Radboud University Nijmegen, The Netherlands \\ ${ }^{\mathrm{b}}$ Cognitive Brain Research Unit, Department of Psychology, University of Helsinki, Finland
}

Received 4 April 2005; received in revised form 8 June 2005

Available online 19 August 2005

\begin{abstract}
The purpose of this experiment was to compare components of the human and rat auditory event-related potential (ERP) in a serial feature-positive discrimination task. Subjects learned to respond to an auditory target stimulus when it followed a visual feature $(X \rightarrow A+)$, but to not respond when it was presented alone $(\mathrm{A}-)$. Upon solving the task, the N2 component, which has been suggested to reflect the activation of inhibitory processes, was temporarily more negative in response to the target on $\mathrm{A}-$ than on $\mathrm{X} \rightarrow \mathrm{A}+$ trials in both species. However, whereas a P3 component was present in the human participants, this component was absent in the rats. In both species, the amplitude of several ERP components, including the N2, decreased in the course of training. These results are discussed in the framework of contemporary models of associative learning.
\end{abstract}

(c) 2005 Elsevier Inc. All rights reserved.

Keywords: Auditory event-related potential; Discrimination task; Human; N2; Occasion setting; P3; Rat

\footnotetext{
* Corresponding author. Fax: +31 243616066.

E-mail address: anke.sambeth@cbru.helsinki.fi (A. Sambeth).
} 
Event-related potentials (ERPs) are averaged electrical brain potentials triggered by, and time-locked to, sensory, motor, or cognitive events. These potentials consist of various components that are either defined by polarity and order of occurrence (e.g., the $\mathrm{N} 2$ is the second negative component), or by polarity and latency (e.g., the P300 is a positive component approximately $300 \mathrm{~ms}$ after stimulus onset).

In the human auditory ERP, P50, N100, P200, N200, and P300 (P1, N1, P2, N2, and $\mathrm{P} 3$, respectively) components can be distinguished. One frequently studied ERP component is the P3. It is often elicited in oddball tasks, in which frequent standard stimuli are interspersed with infrequent target stimuli. The P3 has a larger amplitude following target stimuli than following standard stimuli (see, e.g., Ochoa \& Polich, 2000; Sambeth et al., 2003; for recent examples) and is generally larger during relevant than irrelevant stimuli (e.g., Kok, 2001; Sambeth et al., 2003). A further frequently studied component in humans is the N2, which is more negative if a subject has to refrain from responding than if he/she is required to make a response. Traditionally, the $\mathrm{N} 2$ has been suggested to reflect the inhibition of a motor response (Bokura, Yamaguchi, \& Kobayashi, 2001; Bruin \& Wijers, 2002; Falkenstein, Hoormann, \& Hohnsbein, 1999), although more recent studies suggest that it rather reflects a conflict arising from the competition between the execution and inhibition of a single response (Donkers \& Van Boxtel, 2004; Zang, Wang, Li, \& Wang, 2003).

Previous studies have shown P20, N60, P120, and N160 (P1, N1, P2, and N2, respectively) components in rats in response to auditory stimuli (Ehlers, Kaneko, Robledo, \& Lopez, 1994; Meeren, Van Cappellen vanWalsum, Van Luijtelaar, \& Coenen, 2001; Sambeth, Maes, Quian Quiroga, \& Coenen, 2004; Sambeth et al., 2003; Shinba, 1997; Yamaguchi, Globus, \& Knight, 1993). A further component found in several rat studies is interpreted as a P300 (P3) component (e.g., Ehlers et al., 1994; Hurlbut, Lubar, \& Satterfield, 1987; Shinba, 1997, 1999). Fairly recently, ERP studies using rats have also used oddball tasks to assess whether cognitive processes and corresponding ERP components in rats are similar to those in humans (Brankačk, Seidenbecher, \& Müller-Gärtner, 1996; Ehlers et al., 1994; Hurlbut et al., 1987; Sambeth et al., 2003; Shinba, 1997, 1999; Yamaguchi et al., 1993). Some of these studies found a positive component at approximately $300 \mathrm{~ms}$ after stimulus onset (Brankačk et al., 1996; Ehlers et al., 1994; Hurlbut et al., 1987; Shinba, 1997, 1999), which was more positive for targets than for standards. Others (Galicia et al., 2000; Yamaguchi et al., 1993), however, found a component with an earlier latency (220-240 ms). It is not yet clear whether these P3-like components reflect the same cognitive processes as the P3 does in humans (Sambeth et al., 2003).

The present experiment directly compared the ERPs of humans and rats. The ERP components elicited in the rat during oddball tasks are relatively well described, but it is not known whether the same components can be found in these animals in other cognitive tasks, and whether they are as comparable as in the case for the rat and human ERP components during oddball tasks. Therefore, we used a procedure that, in contrast to the oddball paradigm, has extensively been studied behaviourally in rats, but much less in humans, namely a serial feature-positive discrimination procedure. Apart from the inter-species comparison, an additional aim of the present 
study was to examine whether the ERP measure can be a valuable tool to further assess the nature of the processes involved in this kind of task.

In a feature-positive discrimination, a target stimulus (A) is followed by an unconditioned stimulus (US, e.g., food) when it is preceded by a feature stimulus (X), and is not followed by the US when presented alone. Accordingly, subjects have to learn to respond to A only if it is preceded by $\mathrm{X}$. Adequate responding in this task may reflect two different associative structures. First, responding may be based on a direct association between the feature and the US. Second, responding may be based on an association between the target stimulus and the US, the strength of which is modulated by the feature stimulus. The latter associative structure is also known as 'occasion setting,' reflecting that the feature sets the occasion for responding to the target. It has been shown that under relatively well-defined circumstances, such as when there is an empty interval between termination of the feature and onset of the target, it is occasion setting that underlies the performance of both rats and humans rather than a direct association (for human studies see, e.g., Baeyens et al., 2004; Dibbets, Maes, \& Vossen, 2002; Hardwick \& Lipp, 2000; for reviews on animal research see Holland, 1992; Swartzentruber, 1995).

At least two different models have been proposed regarding the nature of the association underlying occasion setting (see Fig. 1). In the model described by Holland (1992), feature X positively activates the excitatory A-US association (see Fig. 1A). The model described by Nelson and Bouton (1997; Bouton \& Nelson, 1998) assumes not only excitatory but also inhibitory activity (see Fig. 1B). However, the feature only affects the inhibitory link. If feature X precedes A, X inhibits the inhibitory link, thereby primarily leaving the excitatory link intact. If $\mathrm{A}$ is presented alone, however, the inhibition process is active and the effect of the excitatory link is attenuated or eliminated. There is still much debate as to the validity of these two, and related, types of models of occasion setting (e.g., Hall, 2002; Swartzentruber, 1995). As will be outlined below, ERP studies might be helpful in evaluating the validity of these different models.

A

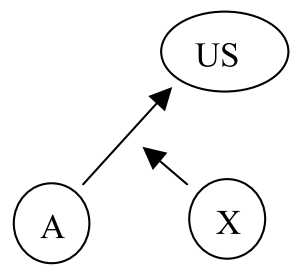

B

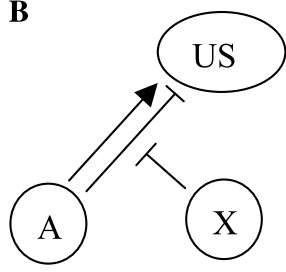

Fig. 1. Models of the associative structure underlying performance in a serial feature-positive discrimination task. A, target; X, feature; US, unconditioned stimulus; arrows indicate an excitatory association; a blocked line indicates an inhibitory association. (A) The model provided by Holland (1992), in which X modulates the excitatory A-US link, leading to more excitation on $\mathrm{X} \rightarrow \mathrm{A}+$ trials compared to $\mathrm{A}-$ trials. (B) The model described by Bouton and Nelson (1998). By presenting X, the inhibitory A-US association is inhibited, thus canceling inhibition, whereas the inhibitory link is present during A-alone presentations, counteracting the excitatory link. 
In the current feature-positive discrimination task, both humans and rats learned to respond to an auditory target when it was preceded by a visual feature $(\mathrm{X} \rightarrow \mathrm{A}+)$ and to not respond when the target was presented alone $(\mathrm{A}-)$. The ERP in response to the target stimulus was analysed for each trial type in both humans and rats. For the human subjects, an additional control experiment was performed to compare ERP responses to the feature between an active condition (feature-positive discrimination task) and a passive condition (only visual stimuli without a need to make a response). As will be outlined in Discussion, this comparison yields valuable information that is relevant to the issue of the nature of the associations underlying task performance. Specifically, it enables an evaluation of the extent to which this feature receives active processing in the course of the experiment.

It is important to note that the stimuli and procedure used to some extent differed between the two species. However, former learning research has shown that different procedures can lead to similar or even identical outcomes in humans and rats (e.g., Dibbets, Maes, \& Vossen, 2000; Dibbets et al., 2002), suggesting that these procedures are successful in provoking identical cognitive processes. To the best of our knowledge, no ERP research has been performed at all in both humans and animals using these kinds of learning tasks. However, based on the above-mentioned models of occasion setting, and earlier results of human ERP research, several working hypotheses can be formulated concerning the N2 and P3 components in the current experiment.

The human N2 component is either related to response inhibition processes (Bokura et al., 2001; Falkenstein et al., 1999) or to a conflict between excitation and inhibition (Donkers \& Van Boxtel, 2004). According to the model of Nelson and Bouton (1997; Bouton \& Nelson, 1998), modulation of responding to target A is realized by means of the on- or offset of an inhibitory link. If feature $\mathrm{X}$ is present, the inhibitory A-US link is weakened or eliminated; if $\mathrm{X}$ is not present the inhibitory link is fully present. Therefore, if the N2 indeed reflects the presence of an inhibitory process, this component might be expected to be more negative (more pronounced) during $\mathrm{A}-$ than $\mathrm{X} \rightarrow \mathrm{A}+$ trials. Alternatively, the two trial types should not elicit a differential $\mathrm{N} 2$ response if the modulation is accomplished through an excitatory effect, as in Holland's model.

As, to our knowledge, response inhibition in relation to ERPs has not been studied in rats before, we cannot formulate a clear hypothesis about whether this effect will be present, and if so, at which ERP component.

The human P3 component is more positive during relevant than during irrelevant stimuli. Furthermore, some authors (e.g., Johnson, 1986; Kok, 2001) proposed that the amplitude of the P3 component is affected by factors such as probability, attention, expectancy, or task relevance. According to the model of Holland (1992), excitation is the key factor in occasion setting, and, therefore, one could hypothesise that the P3 component will be larger in response to the more relevant $\mathrm{X} \rightarrow \mathrm{A}+$ trials than to $\mathrm{A}-$ trials, at least in the human participants. Although P3-like components have been found in rats before, we do not know whether they reflect the same cognitive processes as the P3 component does 
in humans (Sambeth et al., 2003). Therefore, again, as far as the rat subjects are concerned no clear hypotheses can be formulated for this component in the present task.

Given the lack of data on the electrophysiological correlates of feature-positive discrimination learning and performance, no particular hypotheses were formulated about the other human and rat ERP components. However, given the complete lack of ERP data, all empirical results are of importance. For this reason, we recorded and analysed all components. Additionally, we were interested in the time-course of the ERP effects because of potential changes in the processes underlying performance on the present discrimination task. Therefore, the data were offline divided into several blocks.

\section{Methods A, humans}

\section{Subjects}

Eleven students (three men and eight women, mean age 23 years) of the Radboud University Nijmegen, The Netherlands, participated in the feature-positive discrimination task (experimental group). Another 12 participants took part in a control experiment (control group), in which only visual features were presented without the need to make any responses ( 2 men and 10 women, mean age 22 years). All students were either paid for their participation, or received course credits. They were only allowed to take part in the study if they were healthy, did not use medication, and had no psychiatric history. The students who agreed to participate signed an informed consent.

\section{Apparatus}

The participants were tested in a sound-attenuating, dimly lit cubicle (inside dimensions: $2 \times 2.2 \times 2 \mathrm{~m}$ ). The participants were seated in a comfortable chair. A 17 in. computer monitor, which was used to present auditory and visual stimuli, was placed $1.5 \mathrm{~m}$ in front of the participant. A black circle with a radius of $6 \mathrm{~cm}$ served as a visual stimulus for all participants; a $1500-\mathrm{Hz}, 70-\mathrm{dB}(\mathrm{A})$ tone served as an auditory stimulus only for the experimental group. Participants had a response button in their preferred hand during the experiment.

\section{Electrode placement}

In all participants, EEG activity was recorded using an electrode cap with tin electrodes from the $\mathrm{Fz}$ (frontal), $\mathrm{Cz}$ (central), and $\mathrm{Pz}$ (parietal) sites according to the international 10-20 system, with the right mastoid as reference. Eye movements were detected by horizontal and vertical electro-oculogram (EOG) recordings. Impedance was less than $5 \mathrm{k} \Omega$ for all subjects. EEG and EOG were filtered between 0.016 and $100 \mathrm{~Hz}$ and sampled at $512 \mathrm{~Hz}$. 


\section{Procedure experimental group}

First, EEG and EOG electrodes were attached to the skin. Subsequently, the participants were presented a two-block serial feature-positive discrimination task. The visual stimulus was used as feature $(\mathrm{X})$; the auditory stimulus served as target (A). The visual and auditory stimuli had a duration of $1 \mathrm{~s}$. The time between the end of the feature and the onset of the target was $5 \mathrm{~s}$ (ISI). The interval between trials was 10 $15 \mathrm{~s}$ (ITI). Half the trials consisted of a target that was preceded by feature $\mathrm{X}$ $(\mathrm{X} \rightarrow \mathrm{A})$, whereas the other half consisted of a target $(\mathrm{A})$ presented alone. The participants were required to press a button with their preferred hand immediately after termination of the target stimulus on the $\mathrm{X} \rightarrow \mathrm{A}$ trials $(\mathrm{X} \rightarrow \mathrm{A}+)$, but not on targetalone trials $(\mathrm{A}-)$. After each trial, the participants received feedback on the computer screen about their task performance (correct, incorrect). If a participant responded too early, he/she received a 'too fast' notification without any feedback on the correctness of responding. Each block contained 50 trials of each type. The order of the presentation of trial types was random, with the restriction that no more than three trials in succession were of the same type. The participants received a 5-min break between blocks.

Before the experiment, the human participants received instructions about the fact that stimuli were going to be presented and that they had to learn when they had to press or not to press the button. The participants were further told not to press the button during the presentation of a stimulus, but to wait until the stimulus had ended. The participants sat comfortably in their chair during the experiment and were instructed to keep their eyes focused on the monitor and to sit as still as possible.

\section{Procedure control group}

First, EEG and EOG electrodes were attached to the skin. Subsequently, the participants took part in a task in which only the visual feature was used. The stimulus had a duration of $1 \mathrm{~s}$. The interval between the trials was $10-15 \mathrm{~s}$ (ITI).

The participants had to learn to not press the button in response to the feature stimuli.

One hundred trials were presented within one session.

\section{Data analysis}

\section{Behavioural analysis}

The criterion for the task to be considered as learned in the experimental group was correct responding to at least two $\mathrm{X} \rightarrow \mathrm{A}+$ and at least two $\mathrm{A}-$ trials in succession.

\section{EEG analysis}

The EEG was visually checked off-line for EOG activity and other artefacts. Trials with artefacts were excluded from analysis. Also excluded were the ERPs associated 
Table 1

ERP components in response to targets with corresponding latency ranges for the human and rat subjects

\begin{tabular}{lll}
\hline Component & Humans & Rats \\
\hline P1 & P75 $(40-90 \mathrm{~ms})$ & P40 $(32-47 \mathrm{~ms})$ \\
N1 & N115 $(90-140 \mathrm{~ms})$ & N75 $(50-100 \mathrm{~ms})$ \\
P2 & P200 $(160-240 \mathrm{~ms})$ & P130 $(100-155 \mathrm{~ms})$ \\
N2 & N265 $(220-310 \mathrm{~ms})$ & N180 $(165-200 \mathrm{~ms})$ \\
P3 & P305 $(250-360 \mathrm{~ms})$ at Pz & XXX \\
\hline
\end{tabular}

with incorrect responses, that is in the experimental group, no response to a target on $\mathrm{X} \rightarrow \mathrm{A}+$ trials, a response to the target on $\mathrm{A}-$ trials, or a premature response on $\mathrm{X} \rightarrow \mathrm{A}+$ trials. In the control group, incorrect responding was pressing a button in response to the visual stimulus. The EEG fragments within an epoch of $100 \mathrm{~ms}$ before target onset and $500 \mathrm{~ms}$ after target onset were averaged for all correct responses, using the $100 \mathrm{~ms}$ prestimulus as baseline value.

Using the ERP in response to the target, a separate average was determined for each individual of the experimental group and for each of the two trial types. Grand averages were constructed for trial types. Subsequently, the data were divided into four blocks of 25 trials of each type, on the basis of task accomplishment (all participants were able to perform well by the end of Block 1, see Results). However, only the first three of these 25-trial blocks were statistically analysed because the results for Blocks 3 and 4 were almost identical.

For both the experimental and control groups, averages were made for responses to the visual stimuli. These averages were divided into four blocks of 25 trials. As was the case for the responses to the targets, only the first three blocks were used for statistical analysis.

The peak amplitudes of the ERP components were determined on the basis of the individual and grand average ERPs, using the $100 \mathrm{~ms}$ prestimulus as baseline value. Table 1 summarizes the components that were determined for statistical analysis of the ERP data in response to targets. With regard to the visual stimuli, only the P300 component was analysed, which was taken from the same latency as the P3 in response to the targets.

\section{Statistical analysis of the EEG data}

Analyses of variance (ANOVAs) were calculated. For the experimental group, separate analyses were performed for each ERP component with Trial type $(\mathrm{X} \rightarrow \mathrm{A}+$ and $\mathrm{A}-$ ) and Block as within-subject factors, using the ERP's measured as a response to the target stimulus. No ANOVA was performed on the amplitude of the $\mathrm{P} 3$ at $\mathrm{Fz}$ and $\mathrm{Cz}$, because we were uncertain as to whether this was a real $\mathrm{P} 3$ component (it was not visible after Block 1). Subsequently, the P300 response to the visual feature of the experimental and control groups was compared. Block served as a within-subject factor and Group as a between-subject factor in this analysis. The Bonferroni test was used for post hoc analyses. The level of significance was set at .05 throughout. 


\section{Methods B, rats}

\section{Subjects}

Sixteen one-year-old male Wistar rats served as the animal subjects. They were maintained on a 12/12-h light-dark cycle with lights off at 8.00 a.m. The animals were singly housed in Plexiglas cages with unlimited access to water. The rats were kept at $85 \%$ of their free-feeding body weight $(379 \mathrm{~g} \pm 34 \mathrm{~g})$ by restricted daily feeding. Animals were handled daily during the experiment. The study was performed in accordance with the Guidelines of the European Community for the use of experimental animals, and approval was obtained from the Local Ethics Committee.

\section{Apparatus}

The animal subjects were trained and tested in a set of eight identical operant boxes in which EEG recordings could be made. Each box measured $25 \times 24 \times 40 \mathrm{~cm}$. The front and back walls were clear Plexiglas; the right side wall and floor were composed of $3-\mathrm{mm}$ stainless steel rods that were spaced $1.3 \mathrm{~cm}$ apart. The top was left open to enable EEG recordings in the freely moving rat. Centered in the aluminum left side wall was a $5 \times 5 \times 3 \mathrm{~cm}$ recessed food magazine to which 45-mg precision food pellets could be delivered. Visits to the food magazine were registered by means of an infrared emitter and sensor. Two LEDs, which produced a green light that served as a visual feature, were mounted on the left side wall, $6 \mathrm{~cm}$ to the left and to the right, and $3 \mathrm{~cm}$ above the food-magazine. Two speakers were mounted on the left side wall, $6 \mathrm{~cm}$ to the left and to the right of the food-magazine. These speakers were used for presenting a 4-kHz tone with an intensity of $70 \mathrm{~dB}(\mathrm{~A})$ that served as a target stimulus. Each box was enclosed in a sound-attenuating chamber containing a printed circuit board, a set of cables, and a swivel for EEG measurements.

\section{Electrode placement}

A tripolar EEG electrode was implanted epidurally under isoflurane inhalation anaesthesia. The first active lead was inserted near the parietal association cortex (A -3 , 5; L - 2,0 related to bregma) (Paxinos \& Watson, 1998). The second active lead and the ground were placed on the cerebellum. Two screws and dental acrylic cement were employed to fix the electrode on the skull surface. EEG was filtered between 0.1 and $100 \mathrm{~Hz}$ and sampled at $512 \mathrm{~Hz}$.

\section{Procedure}

First, the tripolar EEG electrode was implanted epidurally and the rats were allowed to recover for two weeks. This period was followed by a period of food restriction. Next, the animals received two 30-min magazine training sessions in 
which they learned to retrieve food pellets from the food magazine. Ten food pellets were delivered according to a 3-min variable time schedule in each session. Subsequently, the rats were trained in 12 120-min sessions on a feature-positive discrimination task, in which they learned to visit the pellet feeder during an auditory target when it was preceded by the visual feature $(\mathrm{X} \rightarrow \mathrm{A}+)$, but not when the target was presented alone $(\mathrm{A}-)$. This was achieved by delivering a food pellet after termination of the target on $\mathrm{X} \rightarrow \mathrm{A}$ trials, and not delivering a food pellet after $\mathrm{A}$ alone presentations. For each trial, it was recorded whether a rat visited the pellet feeder. Both the visual and the auditory stimuli had a duration of $5 \mathrm{~s}$ and the ISI was $5 \mathrm{~s}$. The ITI was $1-3 \mathrm{~min}$. Thirty $\mathrm{X} \rightarrow \mathrm{A}+$ and $30 \mathrm{~A}-$ trials were presented each day in a random order, with the restriction that no more than two trials in succession were of the same type.

\section{Data analysis}

\section{Behavioural analysis}

For the rat subjects, a ratio score was calculated by dividing the number of correct responses during targets (magazine visit on $\mathrm{X} \rightarrow \mathrm{A}+$ trials; no visit on $\mathrm{A}-$ trials) by the total number of responses. If the ratio score based on the responses on one training day was larger than 0.7 , the rat had reached the learning criterion.

\section{EEG analysis}

The EEG was visually checked for movement artifacts. Trials with artifacts were excluded from analysis. Also excluded were the ERPs associated with incorrect responses, that is, no response to a target on $\mathrm{X} \rightarrow \mathrm{A}+$ trials, or a response to the target on A- trials. The EEG fragments within an epoch of $100 \mathrm{~ms}$ before target onset and $500 \mathrm{~ms}$ after target onset were averaged for all correct responses, using the $100 \mathrm{~ms}$ prestimulus as baseline value.

Using the ERP in response to the target, a separate average was determined for each rat and trial type. Grand averages were constructed for trial types. To investigate whether the amplitude of the ERP components changed in the course of the experiment, the data were divided into three blocks of four sessions, based on task accomplishment (rats were able to perform well from the start of Block 2, see Results).

The peak amplitudes of the ERP components were determined on the basis of individual and grand average ERPs. Table 1 summarizes the components that were determined for statistical analysis of the ERP data obtained in response to the target stimulus.

\section{Statistical analysis of the EEG data}

Analyses of variance (ANOVAs) were calculated. Separate analyses were performed for each ERP component with Trial type $(\mathrm{X} \rightarrow \mathrm{A}+$ and $\mathrm{A}-)$ and Block as within-subject factors. The Bonferroni test was used for post hoc analyses. The level of significance was set at .05 throughout. 


\section{Results}

\section{Behavioural data of humans and rats: responding to targets}

The human participants reached the learning criterion within the first 10 trials. The mean response time (from target termination to response) across participants was 320,373 , and $350 \mathrm{~ms}$, for Blocks 1, 2, and 3, respectively. Three rats did not reach the learning criterion before the eighth session of the experiment (end of Block 2). They repeatedly responded to $\mathrm{A}-$ trials (false alarms). Therefore, their data were excluded from the analysis. The mean ratio score for the remaining 13 rats was .61 during the first sessions of the experiment (Block 1, learning criterion not reached), and .77 and .81 during Blocks 2 and 3, respectively (learning criterion reached).

\section{Human ERPs in response to targets, experimental group}

Grand average ERPs at Fz, Cz, and Pz of Blocks 1-3 are shown in Figs. 2-4, respectively, as elicited by the target. Five ERP components were discerned: P1, N1, P2, N2, and P3. The mean number of useable trials per participant per block
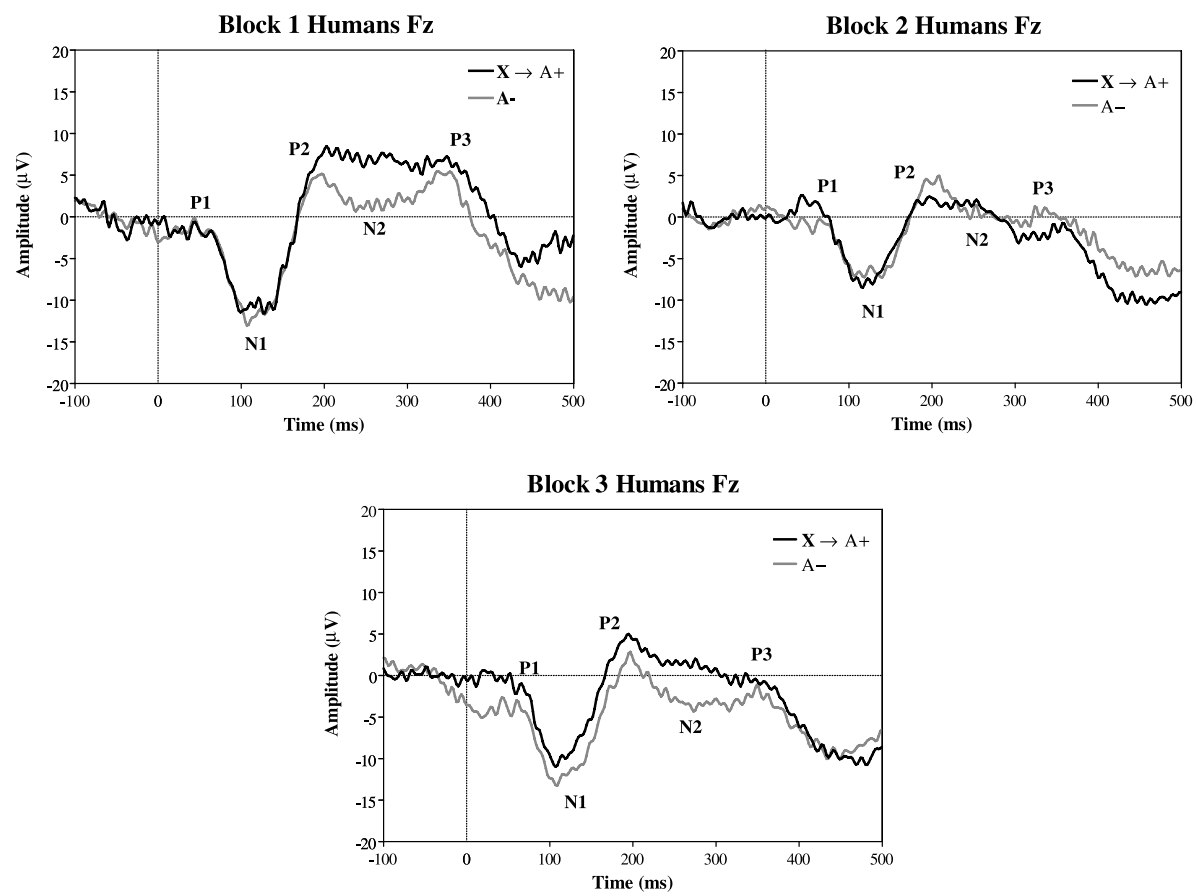

Fig. 2. Grand average ERPs at Fz of the human participants in three blocks of 25 trials, as evoked by targets preceded by a feature $(\mathrm{X} \rightarrow \mathrm{A}+$, black line) and targets presented alone ( $\mathrm{A}-$, grey line). Latencies are shown on the $x$-axis in milliseconds and amplitudes are presented on the $y$-axis in microvolts. Note that, in Block 1, the amplitude of the N2 component was more negative in response to targets presented alone than to targets that were preceded by a feature. 

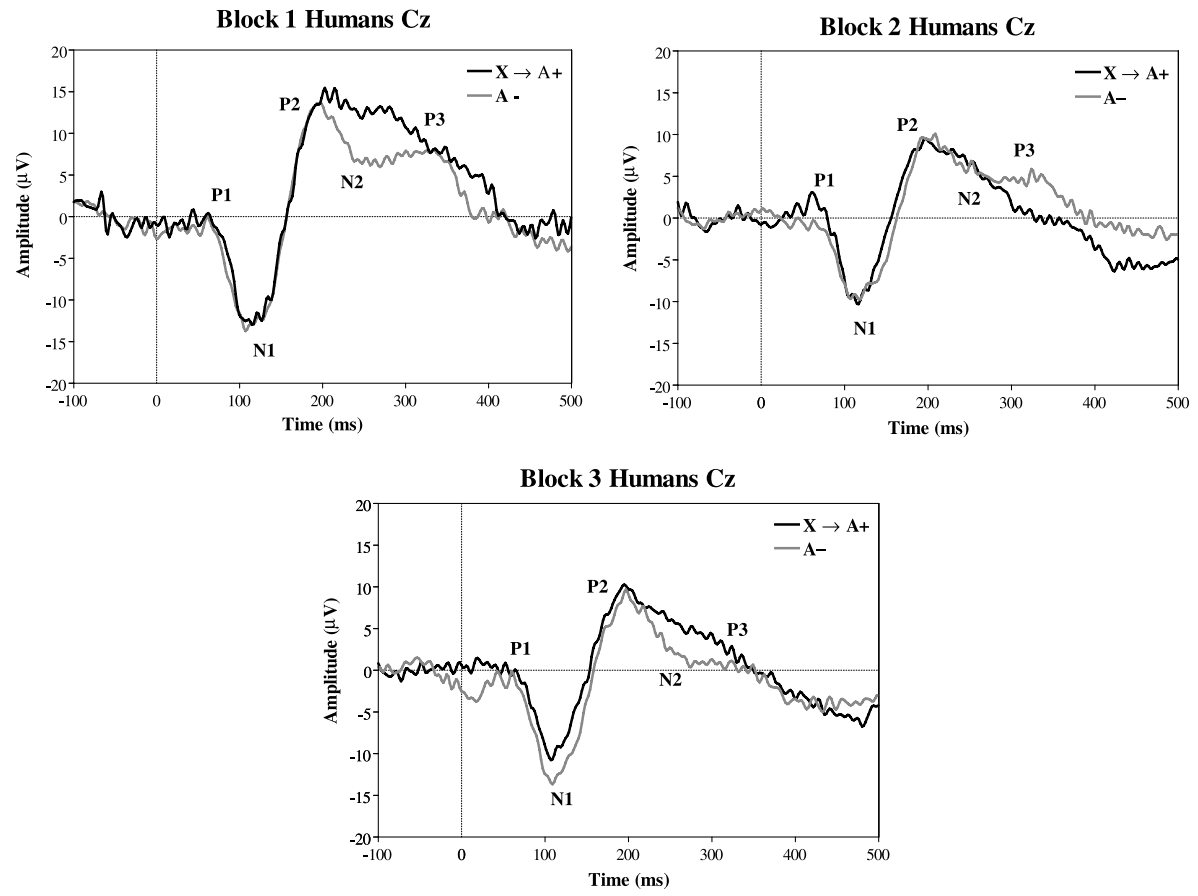

Fig. 3. Grand average ERPs at $\mathrm{Cz}$ of the human participants in three blocks of 25 trials, as evoked by targets preceded by a feature $(\mathrm{X} \rightarrow \mathrm{A}+$, black line) and targets presented alone ( $\mathrm{A}-$, grey line). Note the $\mathrm{N} 2$ effect in Block 1.

calculated over blocks (correct responses and good EEG) was 19 for $\mathrm{X} \rightarrow \mathrm{A}+$ trials and 20 for $\mathrm{A}-$ trials. The figures show that, in Block 1, the amplitude of the N2 component was more negative on $\mathrm{A}-$ trials than on $\mathrm{X} \rightarrow \mathrm{A}+$ trials at all electrodes. Moreover, in Block 1, a $\mathrm{P} 3$ was present at $\mathrm{Pz}$ on $\mathrm{X} \rightarrow \mathrm{A}+$ trials and $\mathrm{A}-$ trials, although this component did not seem to differ in amplitude between trial types at 300-350 ms. In the other blocks, this component had decreased considerably. A final observation is that the amplitudes of the N1, P2, N2, and P3 components decreased in the course of the experiment.

The ANOVA on the amplitudes of the $\mathrm{P} 1$ component revealed a significant main effect of Trial type at Fz, $F(1,10)=5.91, p=.035, \mathrm{Cz}, F(1,10)=6.19, p=.032$, and $\mathrm{Pz}$, $F(1,10)=5.27, p=.045$, but no Block or interaction effects $(F \mathrm{~s} \leqslant 2.60)$. The P1 amplitude was more negative on $\mathrm{A}-$ trials than on $\mathrm{X} \rightarrow \mathrm{A}+$ trials.

An ANOVA on the N1 component amplitude revealed an effect of Block at Fz, $F(2,9)=11.13, p=.004$, and $\mathrm{Cz}, F(2,9)=7.64, p=.011$. No other effects were found with regard to this component at any electrode site $\left(F_{\mathrm{S}} \leqslant 1.54\right)$. Post hoc analysis of the Block effect showed that the N1 was more negative in Block 1 than in Block 2 and also more negative in Block 3 than in Block 2. The analysis on the P2 component revealed a main effect of Block at $\mathrm{Cz}$ only, $F(2,9)=7.93, p=.010$, and no further 

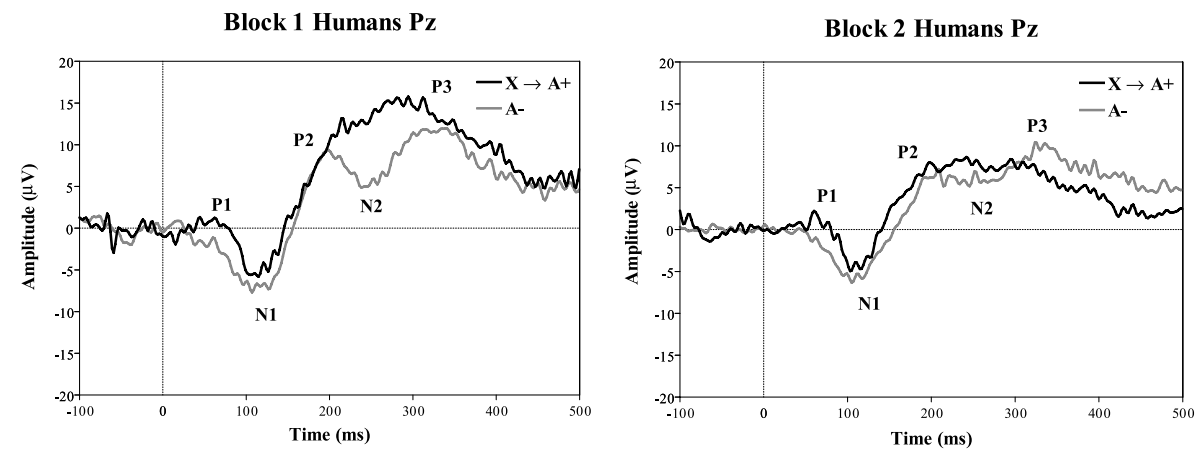

Block 3 Humans Pz

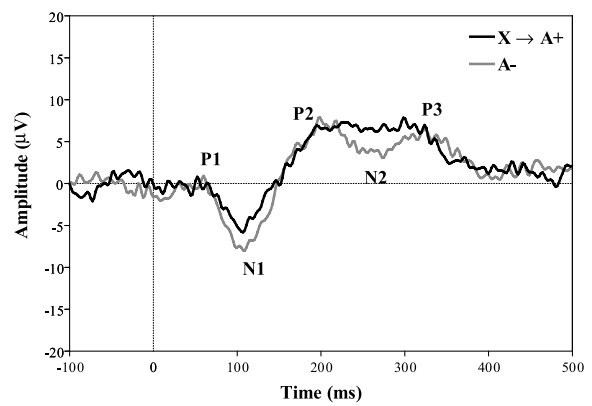

Fig. 4. Grand average ERPs at $\mathrm{Pz}$ of the human participants in three blocks of 25 trials, as evoked by targets preceded by a feature $(\mathrm{X} \rightarrow \mathrm{A}+$, black line) and targets presented alone ( - , grey line). Note the N2 effect in Block 1.

significant effects $\left(F_{\mathrm{S}} \leqslant 3.16\right)$. Post hoc analysis showed that the amplitude of the P2 was more positive in Block 1 than in Block 2.

An ANOVA on the amplitudes of the N2 revealed main effects of Trial type at Fz, $\mathrm{Cz}$, and $\mathrm{Pz},[F(1,10)=5.07, p=.048 ; F(1,10)=8.14, p=.017 ;$ and $F(1,10)=15.59$, $p=.003$, respectively]. No main Block effect or interaction effect was found at either $\mathrm{Cz}$ or $\mathrm{Pz}(F \mathrm{~s} \leqslant 3.72)$, although the interaction at $\mathrm{Fz}$ showed a tendency towards significance, $F(2,9)=3.71, p=.067$. The amplitude of the $\mathrm{N} 2$ was less negative in response to target stimuli on $\mathrm{X} \rightarrow \mathrm{A}+$ trials than on $\mathrm{A}-$ trials. As visual inspection suggested that this was primarily the case for Block 1, we were interested in whether this N2 effect statistically disappeared during the experiment. Therefore, ANOVAs were performed for each block separately. The amplitude of the N2 component proved to be significantly more negative for $\mathrm{A}-$ than for $\mathrm{X} \rightarrow \mathrm{A}+$ trials in Block 1 at $\mathrm{Fz}, F(1,10)=22.80, p=.001, \mathrm{Cz}, F(1,10)=8.27, p=.017$, and $\mathrm{Pz}, F(1,10)=11.39$, $p=.007$, but not in the other blocks $(F \mathrm{~s} \leqslant 4.47)$.

The ANOVA on the amplitudes of the $\mathrm{P} 3$ component at $\mathrm{Pz}$ revealed a main effect of Block, $F(2,9)=19.98, p<.001$, but no main effect of Trial type $(F=3.13)$ and no interaction $(F=.47)$. Post hoc analysis of the main Block effect showed that the amplitude of the P3 was larger in Block 1 than in Blocks 2 and 3. Visual inspection of 
the Block 1 data suggested that there was a difference in P3 amplitude between trials types. As the P3 component was especially important for one of the hypotheses, ANOVAs were performed for each block separately. However, these analyses did not reveal any significant effects $(F \mathrm{~s} \leqslant 1.79)$.

\section{Rat ERPs in response to targets}

Grand average ERPs of the rats are displayed in Fig. 5. This figure shows that four components, with the same order of polarities as found for the human ERPs, were present 40, 75, 130, and $180 \mathrm{~ms}$ after stimulus onset. No P3 was detected. The mean number of useable trials per rat per block calculated over blocks (correct responses and good EEG) was 93 for $\mathrm{X} \rightarrow \mathrm{A}+$ trials and 66 for $\mathrm{A}-$ trials. The reason for the average of $\mathrm{X} \rightarrow \mathrm{A}+$ trials being based on more trials than was the case for $\mathrm{A}-$ trials is that rats made less errors on these former trials. In Block 2, the N2 (at $180 \mathrm{~ms}$ ) component was more negative in response to the target on $\mathrm{A}-$ trials than on $\mathrm{X} \rightarrow \mathrm{A}+$ trials. Furthermore, the figure shows that the amplitudes of the four ERP components showed a tendency towards baseline $(0 \mu \mathrm{V})$ in the course of the experiment.
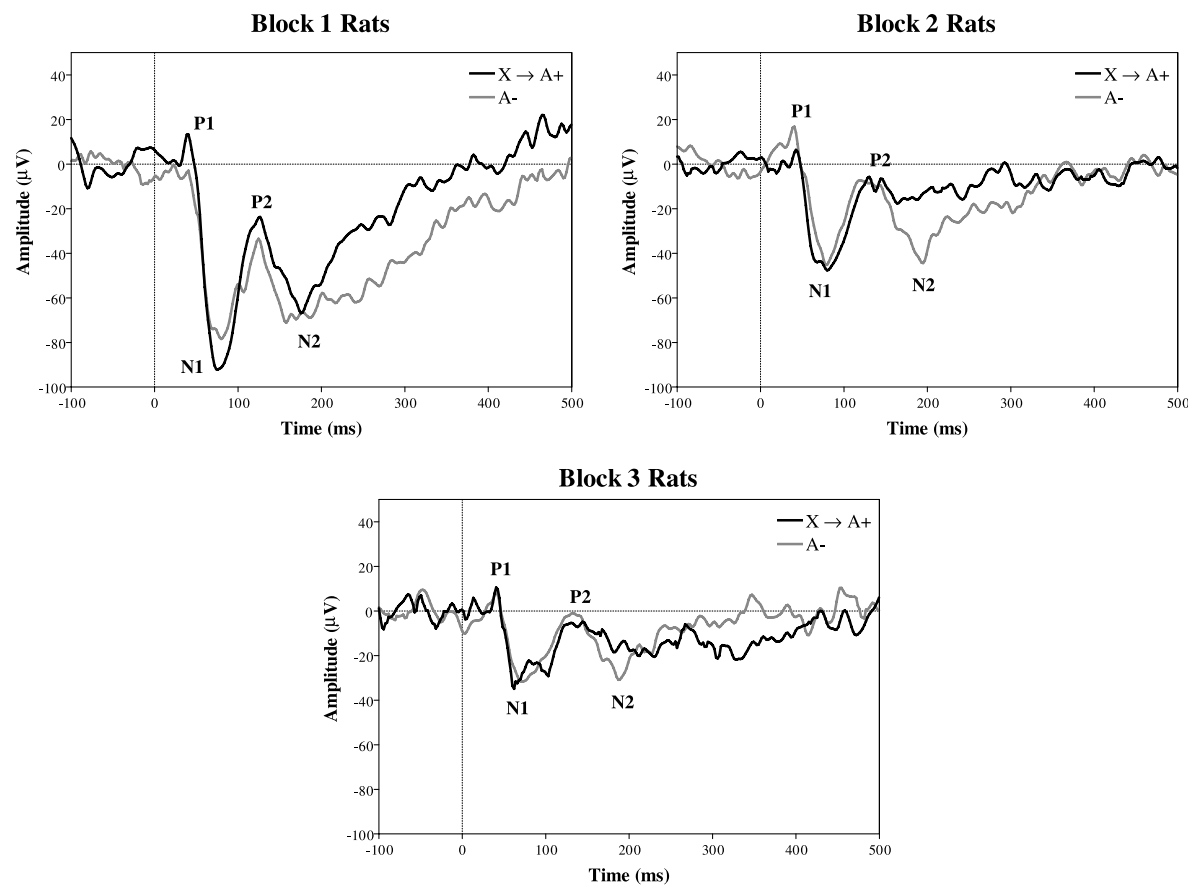

Fig. 5. Grand average ERPs of the rat subjects in three 4-session blocks, as evoked by targets preceded by a feature $(\mathrm{X} \rightarrow \mathrm{A}+$, black line) and targets presented alone ( $\mathrm{A}-$, grey line). Note that, in Block 2, the $\mathrm{N} 2$ amplitude was more negative in response to targets that were presented alone than to targets that were preceded by the feature. 
An ANOVA on the amplitudes of the P1 component did not reveal any significant effects $(F \mathrm{~S} \leqslant 1.65)$. The ANOVA for the N1 component, however, did detect a main effect of Block, $F(2,11)=14.40, p=.001$. No significant main effect of Trial type and no interaction were found with regard to the N1 $(F \mathrm{~s} \leqslant .34)$. Post hoc analysis showed that the amplitude of the N1 was more negative in Block 1 than in Blocks 2 and 3. The ANOVA using the amplitude of the $\mathrm{P} 2$ revealed a main effect of Block, $F(2,11)=4.43, p=.039$, but no other significant effects $(F \mathrm{~s} \leqslant .31)$. The amplitude of this component was more positive in Block 3 than in Block 1.

The ANOVA on the N2 component showed main effects of Trial type, $F(1,12)=5.54, p=.036$, and Block, $F(2,11)=14.01, p=.001$, but no interaction $(F=1.05)$. The amplitude of the $\mathrm{N} 2$ component was more negative in response to the target on $\mathrm{A}-$ than on $\mathrm{X} \rightarrow \mathrm{A}+$ trials. Visual inspection suggested that the effect was only present in Block 2. Therefore, ANOVAs were performed for each block separately, which confirmed this impression $(F(1,12)=5.52, p=.037$ for Block 2 and $F=.51$ and 2.60 for Blocks 1 and 3, respectively). Post hoc analysis of the significant block effect showed that the $\mathrm{N} 2$ was more negative in Block 1 than in Blocks 2 and 3.

\section{Human ERPs in response to feature: experimental and control group}

Grand average ERPs in response to the visual feature of both the experimental and the control groups are shown in Fig. 6, for each of Blocks 1-3. It can be seen that the $\mathrm{P} 3$ component is observable at $\mathrm{Cz}$ and $\mathrm{Pz}$ in the control group, whereas it is only clearly present at $\mathrm{Pz}$ in the experimental group. Therefore, statistical analysis was performed for the P3 at Pz only. Furthermore, it can be seen that the amplitude of the P3 component was equally positive in all three blocks in the experimental group. In the control group, on the other hand, the amplitude of the P3 component was more positive in Block 1 compared to the other two blocks.

The ANOVA on the P3 amplitude at Pz revealed a significant effect of Block, $F(2,20)=5.78, \quad p=.010$, and an interaction between Block and Group, $F(2,20)=4.71, p=.021$. No main effect of group was found $(F=0.005)$. To unravel the significant interaction, ANOVAs were performed for each group separately. No significant effect of Block was present in the experimental group $(F=0.593)$, whereas it was in the control group, $F(2,10)=24.79, p<.001$. Post hoc comparisons revealed that the amplitudes were larger in Block 1 than in Block $3(p<.05)$. Marginally larger amplitudes were elicited in Block 1 than in Block $2(p=.071)$, and in Block 2 compared to Block 3 ( $p=.070)$. In sum, the amplitude of the P3 decreased in the course of the experiment for the control group, whereas it remained the same for the experimental group.

\section{Discussion}

The aim of the present experiment was to compare ERP components of rats and humans that were subjected to an analogous serial feature-positive discrimination task. More specifically, on the basis of the inhibition hypothesis of the N2 and the 

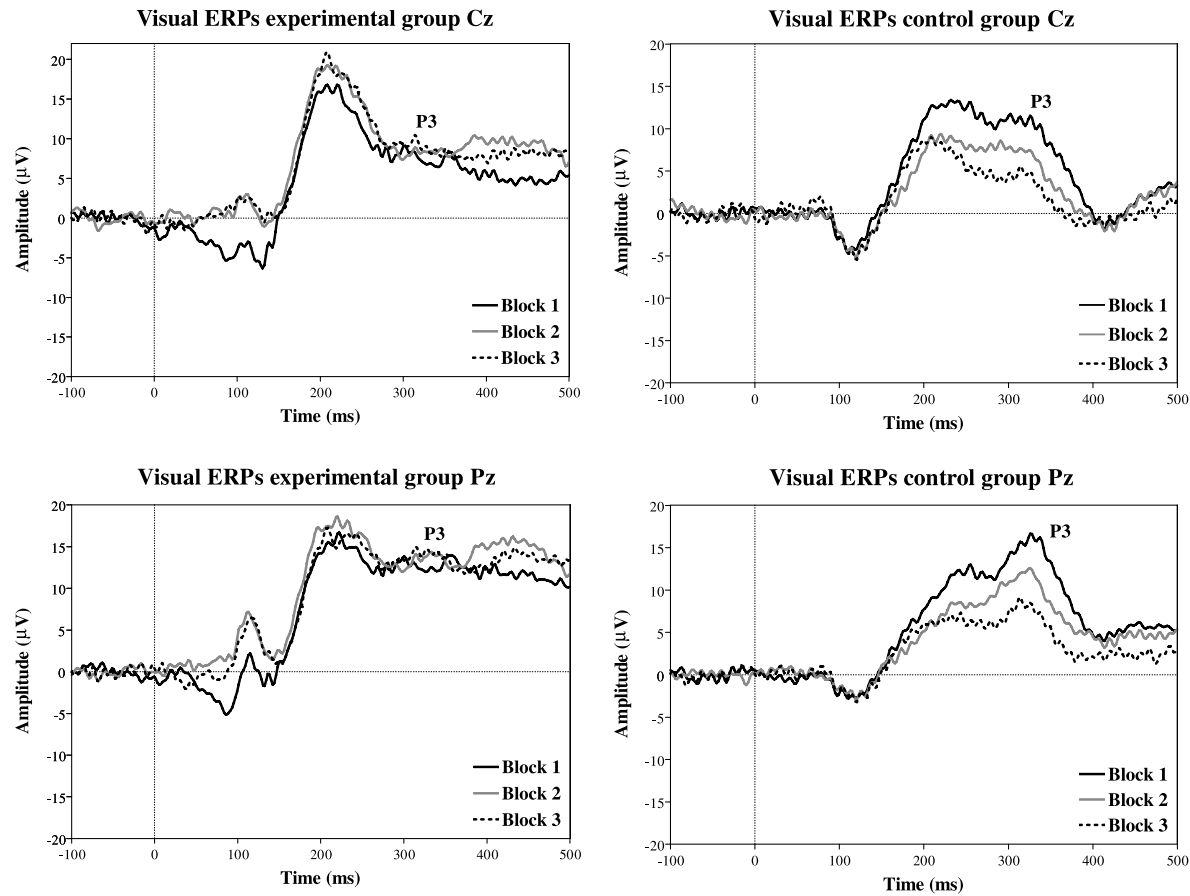

Fig. 6. Grand average ERPs in response to the visual features of the $\mathrm{Cz}$ and $\mathrm{Pz}$ sites of the experimental (left) and control (right) groups, separated for trial Blocks 1-3. Latencies are shown on the $x$-axis in milliseconds and amplitudes are presented on the $y$-axis in microvolts. Note that a large P3 component is observable in the control group, which decreased in amplitude between blocks. In the experimental group, only a large amplitude around $300 \mathrm{~ms}$ is present at Pz. This remains fairly stable in the course of the experiment.

excitation hypothesis of the $\mathrm{P} 3$ components, we were interested in effects on the amplitude of these components. The auditory ERPs elicited by the target stimulus in this task were characterized by the presence of two positive and two negative waves in both humans and rats, albeit with different latencies in the two species. Additionally, a P3 component was present in humans. The N2 component showed analogous task effects in the two species. For both species, the amplitude of this component was more negative on $\mathrm{A}-$ trials than on $\mathrm{X} \rightarrow \mathrm{A}+$ trials on the first block of trials on which the subjects had solved the discrimination task. Besides a differential P1 in humans (for which we do not have an explanation), no other significant task effects were found in either specie. However, regarding the changes in the ERP in the course of the experiment, we again found analogous effects in the two species. The overall shape of the ERP remained the same, but the amplitudes of several components (N1, P2, and P3 in humans; N1, P2, and $\mathrm{N} 2$ in rats) became closer to baseline $(0 \mu \mathrm{V})$ with continued training.

In humans, the response to the visual feature in the feature-positive discrimination task (experimental group) was compared to that in a control group, in which only the visual stimulus was presented without the need to respond. It was shown that, whereas the amplitudes of the P3 component remained stable during the 
feature-discrimination condition, the P3 in the control condition decreased in the course of the experiment. The possible implication of this effect will be addressed later in discussion.

The results of numerous previous studies suggest that, when features and targets are presented serially in a feature-positive discrimination task, the performance of both humans and rats reflects occasion setting. Assuming that the temporal parameters used in our study also evoked occasion setting, we hypothesized that, if the model of Bouton and Nelson (1998) is correct, the N2 component in humans should be more negative for targets on $\mathrm{A}-$ trials than $\mathrm{X} \rightarrow \mathrm{A}+$ trials, which was indeed the case. Furthermore, this effect was most prominent in the first block that the participants had learned the task, and disappeared in the course of the experiment. The N2 component has previously been related to response inhibition (Bokura et al., 2001; Bruin \& Wijers, 2002; Falkenstein et al., 1999), using studies in which participants were instructed to refrain from responding to one kind of stimulus (inhibition) and to press a button to another stimulus type. One important difference with the present study is the fact that participants knew which stimuli were relevant at the start of the experiment in the previous studies, whereas in our study the participants had to learn the relevance of the stimuli during the task. Furthermore, in the previous studies, stimuli requiring no response elicited a large N2 especially in the case of rather low stimulus probabilities (e.g., Bokura et al., 2001). In our study, the corresponding stimuli were presented with a probability of $50 \%$. However, given the fact that, at least in the initial phase of our experiment, we did find equal N2 effects as found in the previous N2 studies, it is not unlikely that the $\mathrm{N} 2$ elicited in our task is at least related to the commonly found $\mathrm{N} 2$ component, both reflecting some kind of response inhibition.

As, to our knowledge, response inhibition has not been explicitly investigated in animal ERP research until now, we did not have a clear hypothesis regarding this issue. However, we found that the rat $\mathrm{N} 2$ component was similarly affected by the task manipulation as was the human N2. It was more negative at $\mathrm{A}-$ trials than at $\mathrm{X} \rightarrow \mathrm{A}+$ trials, and it was most prominent in the block that the rats had learned the task, Block 2. The most parsimonious explanation of these findings is that, if the human N2 indeed is related to response inhibition, the rat N2 component is related to a response inhibition process as well.

Based on Holland's model (1992), the hypothesis concerning the human P3 component was that its amplitude should be larger on $\mathrm{X} \rightarrow \mathrm{A}+$ trials than $\mathrm{A}-$ trials. In the current study, we could identify a P3 component at Pz, but this component did not show any significant task effects, which suggests that the model is incorrect. However, in so-called continuous performance tests (CPT), which are similar to our feature-positive discrimination procedure, it has been shown that the amplitude of the P3 component is more positive on trials requiring a response than on those that do not (Bokura et al., 2001; Tekok-Kilic, Shucard, \& Shucard, 2001). One major difference between our task and the usual CPT paradigms is that we did not instruct our participants how to respond during the task, although this is common practise in human studies. Possibly, instructing participants to especially pay attention to a relevant stimulus (a stimulus that requires responding) results in the corresponding P3 having a higher amplitude than does the $\mathrm{P} 3$ elicited by less relevant stimuli (stimuli that do not require 
responding). Perhaps all target presentations were seen as equally relevant to the participants, leading to an equally large P3 amplitude on the two trial types.

P3-like components have been found in rats during oddball tasks (Brankačk et al., 1996; Ehlers et al., 1994; Hurlbut et al., 1987; Sambeth et al., 2003; Shinba, 1997, 1999). Therefore, we expected to find this component in the feature-positive discrimination task as well, which was not the case. The reason for this absence may be as follows. Human research has shown that the amplitude of the P3 component depends on several factors. Two important factors are probability and interval between stimuli (e.g., Gonsalvez et al., 1999; Katayama \& Polich, 1996; Polich, Ellerson, \& Cohen, 1996; Sambeth, Brankačk, \& Maes, 2004). Other features are, for example, relevance of a stimulus, expectancy, task difficulty, and task complexity (Donchin, 1981; Johnson, 1986; Kok, 2001). One possibility is that, in rats, the P3 amplitude is mostly determined by probability and inter-stimulus interval (external factors), whereas 'cognitive' factors (internal factors) contribute much less to that amplitude. If this were the case, the P3 observed in rats during oddball tasks might be especially due to external factors, whereas internal factors are either not present, or occur at another point in time. In this respect, it is important to note that Talnov, Quian Quiroga, Meier, Matsumoto, and Brankačk (2003), using oddball and single-stimulus tasks, found a negative potential at $150 \mathrm{~ms}$ after stimulus onset in the rat hippocampus only at long inter-stimulus intervals and low probability targets. This negative potential was entirely absent at short intervals and high probability targets. It is, therefore, entirely plausible that the cortically visible P3 in rats behaves in similar ways and was absent in the present study as a result of equal stimulus probabilities.

Based on the current joint results, and if the N2 component found in the present study indeed reflects response inhibition, one could conclude that Bouton and Nelson's inhibition model is more suitable than is Holland's excitation model. 'Excitation' (as reflected in the P3) is present on both trial types (at least for humans), and it is the modulation of inhibition (as reflected in the N2) that is responsible for the differential responding on the two trial types. However, one important qualification must be made: contrary to previous N2 studies, the N2 task effect was only temporary in both species. This can reflect at least one of two mechanisms. A first possibility is that the process underlying the subjects' performance changed from occasion setting to a simple, direct feature-response association in the course of training. Indeed, at least at a behavioural level, many previous animal studies have demonstrated changes in the associative structure underlying task performance as a function of amount of training. Generally, these changes imply a shift from relatively 'rich' associative structures (e.g., those explicitly incorporating a representation of the US used) to more simple associations (such as simple stimulus-response associations) (e.g., see Holland, 2004, for a recent example). At an electrophysiological level, several ERP amplitudes have been found to decrease in both humans and rats in case a stimulus is repeatedly presented and does not require immediate attention and/or action (e.g., Quian Quiroga \& Van Luijtelaar, 2002; Ravden \& Polich, 1999; Sambeth, Maes, Quian Quiroga, Coenen et al., 2004). This decrement, which reflects learning, is very large for the P3 component (Sambeth, Maes, Quian Quiroga, Van Rijn, \& Coenen, 2004). If a stimulus does need attention, the amplitude of the ERP compo- 
nents remains fairly stable. If the feature in the current study continuously acted as an occasion setter, it presumably eventually did not receive much attention, with a corresponding similar decreasing P3 amplitude as was observed for the irrelevant visual stimulus in the control group. This is because, in the experimental group, the importance of the feature stimulus is apparent only at the time of the presentation of the auditory target. If, however, the feature did not continue to act as an occasion setter, but became part of a direct association, the assumption is that attention was increasingly directed at the feature in order to know when to respond. Consequently, the amplitude of the ERP components would not be expected to show a similar decrement as observed in the control group. An examination of the P3 amplitude in response to the visual feature provides evidence in favour of the latter suggestion: this amplitude remained stable throughout the serial feature-positive discrimination task, whereas it decreased in the control condition. This pattern of results indeed is consistent with the notion of a change in underlying mechanism from occasion setting to a direct association between the feature and responding.

A second, related, possibility is that the subjects' performance became more 'automatic' or 'implicit' in the course of training, with implicit processes being governed more by sub-cortical neuronal structures that no longer yielded a strong cortical signal. Evidence for this more general, non-specific, change in the amplitude of several ERP components of both humans (N1, P2, and P3) and rats (N1, P2, and N2) is obtained by the observed tendency towards baseline $(0 \mu \mathrm{V})$. Hence, the ERPs of the two species behaved similarly during the task. The amplitude of ERP components in both humans (e.g., N100, P200, and P300) and rats (e.g., P17, N40) have been found to decrease with repeated presentations in many studies (e.g., Bourbon, Will, Gary, \& Papanicolaou, 1987; Bruin, Kenemans, Verbaten, \& Van der Heijden, 2000; De Bruin et al., 2001; Quian Quiroga \& Van Luijtelaar, 2002; Ravden \& Polich, 1999; Sambeth et al., 2004).

In conclusion, the present study revealed several remarkable similarities with regard to the ERP components of humans and rats in a feature-positive discrimination task, specifically for the N2 component. These results, in turn, suggest strong similarities in cognitive processes in rats and humans, as was also found in previous studies (Sambeth et al., 2003, 2004) and further illustrate how ERP research can be helpful in unravelling the nature of cognitive processes elicited by these kinds of tasks.

\section{Acknowledgments}

We thank Hans Krijnen, Saskia Hermeling, Gerard van Oijen, and Hubert Voogd for their valuable support.

\section{References}

Baeyens, F., Vervliet, B., Vansteenwegen, D., Beckers, T., Hermans, D., \& Eelen, P. (2004). Simultaneous and sequential feature negative discriminations: Elemental learning and occasion setting in human Pavlovian conditioning. Learning and Motivation, 35, 136-166. 
Bokura, H., Yamaguchi, S., \& Kobayashi, S. (2001). Electrophysiological correlates for response inhibition in a Go/NoGo task. Clinical Neurophysiology, 112, 2224-2232.

Bourbon, W. T., Will, K. W., Gary, H. E., \& Papanicolaou, A. C. (1987). Habituation of auditory eventrelated potentials: A comparison of self-initiated and automated stimulus trains. Electroencephalography and Clinical Neurophysiology, 66, 160-166.

Bouton, M. E., \& Nelson, J. B. (1998). Mechanisms of feature-positive and feature-negative discrimination learning in an appetitive conditioning paradigm. In N. A. Schmajuk \& P. C. Holland (Eds.), Occasion setting: Associative learning and cognition in animals. Washington: American Psychological Association.

Brankačk, J., Seidenbecher, T., \& Müller-Gärtner, H.-W. (1996). Task-relevant late positive component in rats: Is it related to hippocampal theta rhythm?. Hippocampus, 6, 475-482.

Bruin, K. J., \& Wijers, A. A. (2002). Inhibition, response mode, and stimulus probability: A comparative event-related potential study. Clinical Neurophysiology, 113, 1172-1182.

Bruin, K. J., Kenemans, J. L., Verbaten, M. N., \& Van der Heijden, A. H. C. (2000). Habituation: An eventrelated potential and dipole source analysis study. International Journal of Psychophysiology, 36, 199-209.

De Bruin, N. M. W. J., Ellenbroek, B. A., Van Schaijk, W. J., Cools, A. R., Coenen, A. M. L., \& Van Luijtelaar, E. L. J. M. (2001). Sensory gating of auditory evoked potentials in rats: Effects of repetitive stimulation and the interstimulus interval. Biological Psychology, 55, 195-213.

Dibbets, P., Maes, J. H. R., \& Vossen, J. M. H. (2000). Interaction between positional but not between nonpositional cues in human predictive learning. Behavioural Processes, 50, 65-78.

Dibbets, P., Maes, J. H. R., \& Vossen, J. M. H. (2002). Serial and simultaneous feature positive discriminations in a human Skinner box. Behavioral Processes, 59, 1-14.

Donchin, E. (1981). Surprise! ... Surprise?. Psychophysiology, 18, 493-513.

Donkers, F. C. L., \& Van Boxtel, G. J. M. (2004). The N2 in go/no-go tasks reflects conflict monitoring not response inhibition. Brain and Cognition, 56, 165-176.

Ehlers, C. L., Kaneko, W. M., Robledo, P., \& Lopez, A. L. (1994). Long-latency event-related potentials in rats: Effects of task and stimulus parameters. Neuroscience, 62, 759-769.

Falkenstein, M., Hoormann, J., \& Hohnsbein, J. (1999). ERP components in Go/NoGo tasks and their relation to inhibition. Acta Psychologica, 101, 267-291.

Galicia, O., Sánchez-Alavez, M., Díaz-Ruíz, O., Sánchez Narváez, F., Elder, J. H., \& Navarro, L. (2000). HIV-derived protein gp120 suppresses P3 potential in rats: Potential implications in HIV-associated dementia. Cognitive Neuroscience, 11, 1351-1355.

Gonsalvez, C. J., Gordon, E., Grayson, S., Barry, R. J., Lazzaro, I., \& Bahramali, H. (1999). Is the target-totarget interval a critical determinant of $\mathrm{P} 3$ amplitude?. Psychophysiology, 36, 643-654.

Hall, G. (2002). Associative structures in pavlovian and instrumental conditioning. In H. E. Pashler \& R. Gallistel (Eds.), Stevens' handbook of experimental psychology: Learning, motivation, and emotion (pp. 1-45). New York: Wiley.

Hardwick, S. A., \& Lipp, O. V. (2000). Modulation of affective learning: An occasion for evaluative conditioning?. Learning and Motivation, 31, 251-271.

Holland, P. C. (1992). Occasion setting and pavlovian conditioning. In D. Medin (Ed.), The Psychology of Learning and Motivation (3rd ed., pp. 69-125). San Diego, CA: Academic Press.

Holland, P. C. (2004). Relations between Pavlovian-Instrumental transfer and reinforcer devaluation. Journal of Experimental Psychology: Animal Behavior Processes, 30, 104-117.

Hurlbut, B. J., Lubar, J. F., \& Satterfield, S. M. (1987). Auditory elicitation of the P300 event-related evoked potential in the rat. Physiology and Behavior, 39, 483-487.

Johnson, R., Jr. (1986). A triarchic model of P300 amplitude. Psychophysiology, 23, 367-383.

Katayama, J., \& Polich, J. (1996). P300, probability, and the three-tone paradigm. Electroencephalography and Clinical Neurophysiology, 100, 555-562.

Kok, A. (2001). On the utility of P3 amplitude as a measure of processing capacity. Psychophysiology, 38, 557-577.

Meeren, H. K. M., Van Cappellen vanWalsum, A. M., Van Luijtelaar, E. L. J. M., \& Coenen, A. M. L. (2001). Auditory evoked potentials from auditory cortex, medial geniculate nucleus, and inferior 
colliculus during sleep-wake states and spike-wave discharges in the WAG/Rij Rat. Brain Research, 898, 321-331.

Nelson, J. B., \& Bouton, M. E. (1997). The effects of a context switch following serial and simultaneous feature-negative discriminations. Learning and Motivation, 28, 56-84.

Ochoa, C. J., \& Polich, J. (2000). P300 and blink instructions. Clinical Neurophysiology, 111, 93-98.

Paxinos, G., \& Watson, C. (1998). The rat brain in stereotaxic coordinates. San Diego: Academic Press.

Polich, J., Ellerson, P. C., \& Cohen, J. (1996). P300, stimulus intensity, modality, and probability. International Journal of Psychophysiology, 23, 55-62.

Quian Quiroga, R., \& Van Luijtelaar, E. L. J. M. (2002). Habituation and sensitization in rat auditory evoked potentials: A single-trial analysis with wavelet denoising. International Journal of Psychophysiology, 43, 141-153.

Ravden, D., \& Polich, J. (1999). On P300 measurement stability: Habituation, intra-trial block variation, and ultradian rhythms. Biological Psychology, 51, 59-76.

Sambeth, A., Brankačk, J., \& Maes, J. H. R. (2004). With long intervals, inter-stimulus interval is the critical determinant of the human P300 amplitude. Neuroscience Letters, 359, 143-146.

Sambeth, A., Maes, J. H. R., Quian Quiroga, R., \& Coenen, A. M. L. (2004). Effects of stimulus repetitions on the event-related potential of humans and rats. International Journal of Psychophysiology, 53, 197205.

Sambeth, A., Maes, J. H. R., Quian Quiroga, R., Van Rijn, C. M., \& Coenen, A. M. L. (2004). Enhanced rehabituation of the orienting response of the human event-related potential. Neuroscience Letters, 356, 103-106.

Sambeth, A., Maes, J. H. R., Van Luijtelaar, G., Molenkamp, I. B. S., Jongsma, M. L. A., \& Van Rijn, C. M. (2003). Auditory event-related potentials in humans and rats: Effects of task manipulation. Psychophysiology, 40, 60-68.

Shinba, T. (1997). Event-related potentials of the rat during active and passive auditory oddball paradigms. Electroencephalography and Clinical Neurophysiology, 104, 447-452.

Shinba, T. (1999). Neuronal firing activity in the dorsal hippocampus during the auditory discrimination oddball task in awake rats: Relation to event-related potential generation. Cognitive Brain Research, 8 , $241-250$.

Swartzentruber, D. (1995). Modulatory mechanisms in Pavlovian conditioning. Animal Learning and Behavior, 23, 123-143.

Talnov, A. M., Quian Quiroga, R., Meier, M., Matsumoto, G., \& Brankačk, J. (2003). Entorhinal inputs to dentate gyrus are activated mainly by conditioned events with long time intervals. Hippocampus, 13, $755-765$.

Tekok-Kilic, A., Shucard, J. L., \& Shucard, D. W. (2001). Stimulus modality and Go/NoGo effects on P3 during parallel visual and auditory continuous performance tasks. Psychophysiology, 38, 578-589.

Yamaguchi, S., Globus, H., \& Knight, R. T. (1993). P3-like potential in rats. Electroencephalography and Clinical Neurophysiology, 88, 151-154.

Zang, X., Wang, Y., Li, S., \& Wang, L. (2003). Event-related potential N270, a negative component to identification of conflict monitoring following memory retrieval. Clinical Neurophysiology, 114, 2461-2468. 